\title{
Biosensors in Applications
}

\section{Kafashan $\mathbf{J}^{1 *}$ and Azadshahraki $\mathrm{F}^{2}$}

${ }^{1}$ Department of Mechanical Engineering in Agro Machinery \& Mechanization, Agricultural Engineering Research Institute, Agricultural Research Education and Extension Organization (AREEO), Karaj, Iran

${ }^{2}$ Research Department of Greenhouse Engineering, Agricultural Engineering Research Institute, Agricultural Research Education and Extension Organization (AREEO), Karaj, Iran

These days, especially, the attention is increasing in high technology of sensors designed for various uses in biological matter, biomedicine, drug improvement, security, nutrition safety and ecological monitoring and measuring. The system of these sensors should typically be handy and economical, on condition that quick response with reliability, high sensitivity, and negligible false-reading counts are provided [1].

Biosensors have a multi-disciplinary nature including: biology, chemistry, physics, instrumentation, electronics, optics, and market. The biosensors are detecting devices that combine a transducer with biologically sensitive and selective components. Biosensors can quantity combinations existing in the environment, chemical procedures, food and human body at inexpensive charge in comparison with traditional techniques [2].

This editorial article briefly focuses on an overview of Biosensors, examples and providing a new vista of developments and applications.

Biosensors have broad prospective applications in such dissimilar domains as screening and monitoring of public and personal health, pathology, environmental monitoring (e.g. the detection of pesticides, river water contaminants, etc.), bioprocess, criminology, civil defense, and within the industry of food, water quality and beverage for safety.

Biosensors are confidently well-known in clinical chemical analysis applications. While different Biosensors in the lab, and in point-ofcare for measurement of blood metabolites such as glucose, lactate, urea and creatinine, using both electrochemical and optical modes of transduction are commercially developed and typically used, difficulties competing with traditional immunoassay based mainly on sensitivity requirements still exist [3].

Progresses in biosensor and biodetection technologies embrace possibilities to modernize healthcare and diagnosis over well-being level, illness initiation and growth evaluations by non-invasive techniques [3,4] (Figure 1).

As can be seen in Figure 1, biosensors normally are made of biorecognition and bio-transducer components, plus an electronic system that includes a signal amplifier, processor, and a display unit. The bio-recognition component, regularly named a bio-receptor, utilizes molecules of bio-receptors modeled after bio-systems to act together

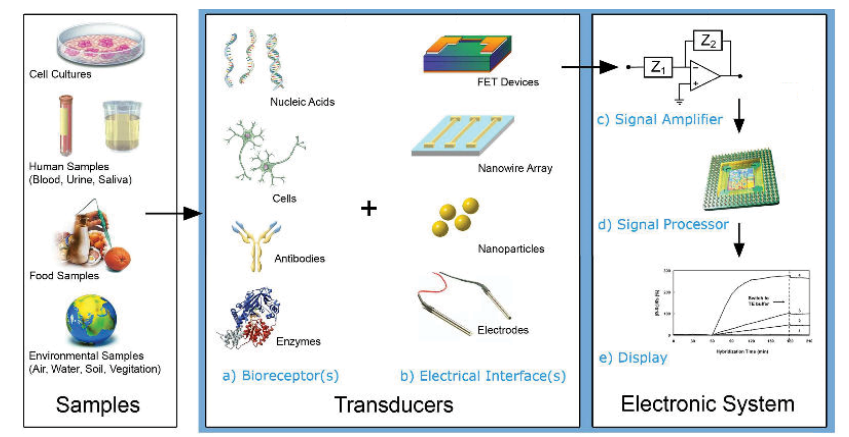

Figure 1: Biosensor-related elements: from samples to electronic systems. with the analyte of concern. This quantifies via the bio-transducer that produces a quantifiable signal related to the level of the objective analyte in the sample. The overall purpose of a biosensor design is to allow swift, appropriate trial at the point of consideration where the samples were obtained [5]. In Figure 2, a scheme of a biosensor system is shown.

Biosensors are generally located in different positions as listed below.

1. In-vivo: In this case, a biosensor takes place inside a body or a living organism.

2. In-vitro: An in-vitro biosensor is one that functions in a culture dish, test tube, or somewhere else outside a living being.

3. At-line: A biosensor is applied in a line of production where a sample can be searched out, analysed, and a conclusion can be reached whether or not the continuation of the production should happen. The monitoring of lactose in a dairy processing plant can be mentioned as an example [6].

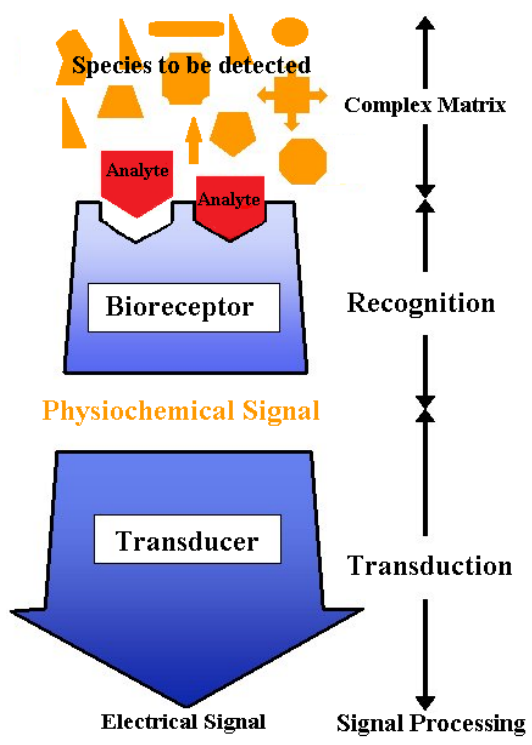

Figure 2: Scheme of a biosensor system.

*Corresponding author: Jalal Kafashan, Department of Mechanical Engineering in Agro Machinery \& Mechanization, Agricultural Engineering Research Institute, Agricultural Research Education and Extension Organization (AREEO), Karaj, Iran, Tel: 016324010; E-mail: jalal.kafashan@kuleuven.be

Received August 04, 2016; Accepted August 06, 2016; Published August 16 , 2016

Citation: Kafashan J, Azadshahraki F (2016) Biosensors in Applications. J Biosens Bioelectron 7: e143. doi:10.4172/2155-6210.1000e143

Copyright: @ 2016 Kafashan J, et al. This is an open-access article distributed under the terms of the Creative Commons Attribution License, which permits unrestricted use, distribution, and reproduction in any medium, provided the original author and source are credited. 


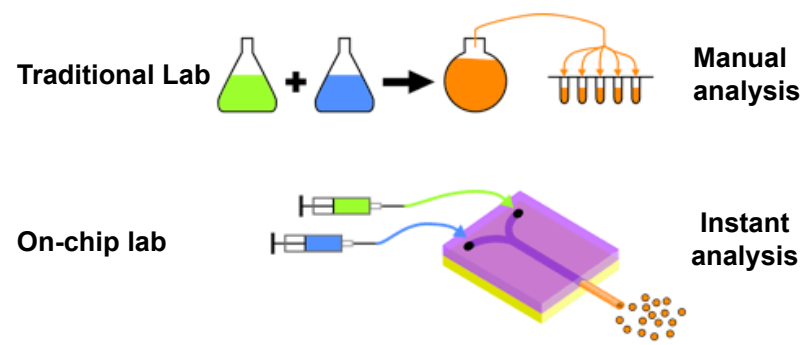

Figure 3: Both processes of a traditional chemical lab and a lab-on-a-chip devices. The lab-on-a-chip device is much smaller and faster than traditional lab methods [8].

4. In-line: An in-line biosensor can be located within a line of production to examine a variable with continuous production, which is possible to be automated fully. The water purification is one of applications of an in-line biosensor.

Point-of-concern: Using a biosensor in the location where the test is needed, which can be taken straight to the "point of concern". An example of this biosensor can be found in testing of blood alcohol content (BAC) from a breath sample by Breathalyser (a portmanteau of breath) that can be implemented at a traffic safety checkpoint.

There are many themes, which are immeasurable easily in Bioengineering and Biomedicine; the use of this technology can be a way to simplify and to get gain of such a progress.

In the next age, it might be imaginable to bring back normal vision and inverse the spinal cord injury and illness effects by a lab-on-a-chip (LOC) device for medical diagnoses with no clinical detection.

Experiment, devices, testing, sensing, monitoring in biomaterials and other steps of a bioprocess for no matter which, can be a great deal with staff, time limitations, facilities, specific conditions and budget [7].
Therefore, an elimination of lab work can speed up the process and can save time and money. The simplicity of a lab-on-a-chip in comparison to a traditional lab is schematically shown in Figure 3.

In conclusion, in the near future, biosensors technology would be a widespread phenomenon in various applications across the food supply chain, pest control, animal production, healthcare, biosecurity, defence, vehicle and transportation, etc.

After all, biosensor would become a common issue in many engineering and non-engineering even not limited to Bioengineering, Biomedicine and other mentioned domains.

The most important future challenges for biosensors consist of: size, remote operation, reliability, sensitivity, accuracy, sample handling, telemetry and range of analytes. All of these issues need to be considered in the designs, implementations and research plans for Biosensors.

\section{References}

1. Magnusson R (2013) The Complete Biosensor. J Biosens Bioelectron 4: e120.

2. Serra PA (2011) New Perspectives in Biosensors Technology and Applications. InTech. pp: 448.

3. Gouvea C (2011) Biosensors for Health Applications. In: PA Serra (ed.) Biosensors for Health, Environment and Biosecurity.

4. Bohunicky B, Mousa SA (2011) Biosensors: The New Wave in Cancer Diagnosis. Nanotechnol Sci Appl 4: 1-10.

5. https://en.wikipedia.org/wiki/Biosensor

6. Glithero N, Clark C, Gorton L, Schuhmann W, Pasco N (2013) At-line Measurement of Lactose in Dairy-Processing Plants. Anal Bioanal Chem 405: 3791-3799.

7. Kafashan J (2013) Computational Simulations: Alternative Solution in Sensing and Monitoring of Biomaterials. J Biosens Bioelectron 4: e118.

8. Brivio M, Verboom W, Reinhoudt DN (2006) Miniaturized Continuous Flow Reaction Vessels: Influence on Chemical Reactions. Lab Chip 6: 329-344. 